\title{
Very Low Energy Electron Transmission Spectroscopy of 2D Materials
}

Ivo Konvalina, Benjamin Daniel, Martin Zouhar, Aleš Paták, Jakub Piňos, Tomáš Radlička, Luděk Frank, Ilona Müllerová and Eliška Materna-Mikmeková

Institute of Scientific Instruments of the CAS, v. v. i., Brno, Jihomoravsky kraj, Czech Republic

Detailed knowledge of mechanisms of electron scattering and its practical consequences for very low energies are of prime importance for not only measurement techniques but also for development of new materials for electronic devices of the next generation. Low thickness of 2D materials motivated us to develop unique device analyzing samples via transmitted electrons in a standard microscopic regime and also via time-of-flight (ToF) spectroscopic method.

The ultra-high vacuum (UHV) scanning low energy electron microscope (SLEEM) equipped with ToF spectrometer (Figure 1) was developed and assembled at Institute of Scientific Instruments (ISI). The UHV SLEEM/ToF system can operate as a standard scanning electron microscope in the range of primary beam energy from $5 \mathrm{keV}$ to units of $\mathrm{eV}$. The microscope is equipped with an electron gun developed by Delong Instruments [1], an in-house built specimen manipulator with biased sample holder for use of the cathode lens (CL) mode [2], and several electron detectors for secondary, backscattered and transmitted electrons.

The new operating mode, the ToF mode, allow us to measure the ToF spectra [3] of electrons transmitted through the sample. The commercial electronics provided with the multi-channel plate (MCP) detector [4] has been complemented by other components.

This includes a pulse generator which allows the microscope to work in a pulse mode enabling the ToF spectrometer functionality. The software supplied to the MCP detector records timestamps of all three types of events, namely signal detections at the MCP, pulse emission towards a given pixel of the sample and pixel switch. These timestamps are used to reconstruct the image of the sample (Figure 1 displaying grayscale intensity of detections for each pixel) and the ToF spectra in the time domain (Figure 2) from the directly measured data, i.e. the timestamps.

Theoretical ray tracing of transmitted electrons allows to find optimal potentials on the electrodes of the ToF spectrometer which shall be used during measurement and in creation of the time-to-energy conversion table. The reconstructed ToF time domain spectrum has been converted to the energy domain (Figure 2). This energy domain spectrum characterizes the electron scattering phenomena in the 2D materials at very low kinetic energies. Key parameter entering propagation of electron in materials is probability of inelastic scattering, which determines the inelastic mean free path (IMFP) [5]. The IMFP can be extracted from the calculated energy loss spectrum (not presented here) using conventional models $[6]$. 

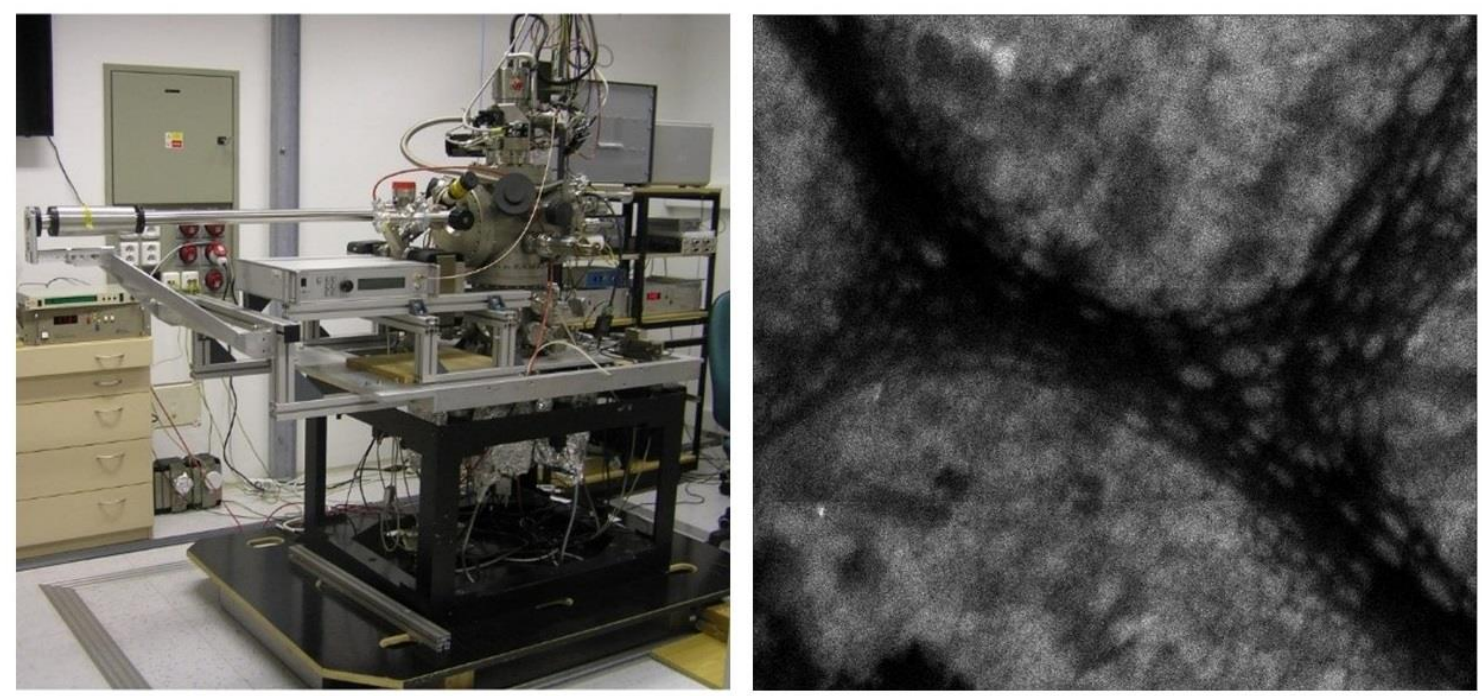

Figure 1. View of the UHV SLEEM/ToF system (left), Transmission image of graphene sample taken by the MCP detector (right).

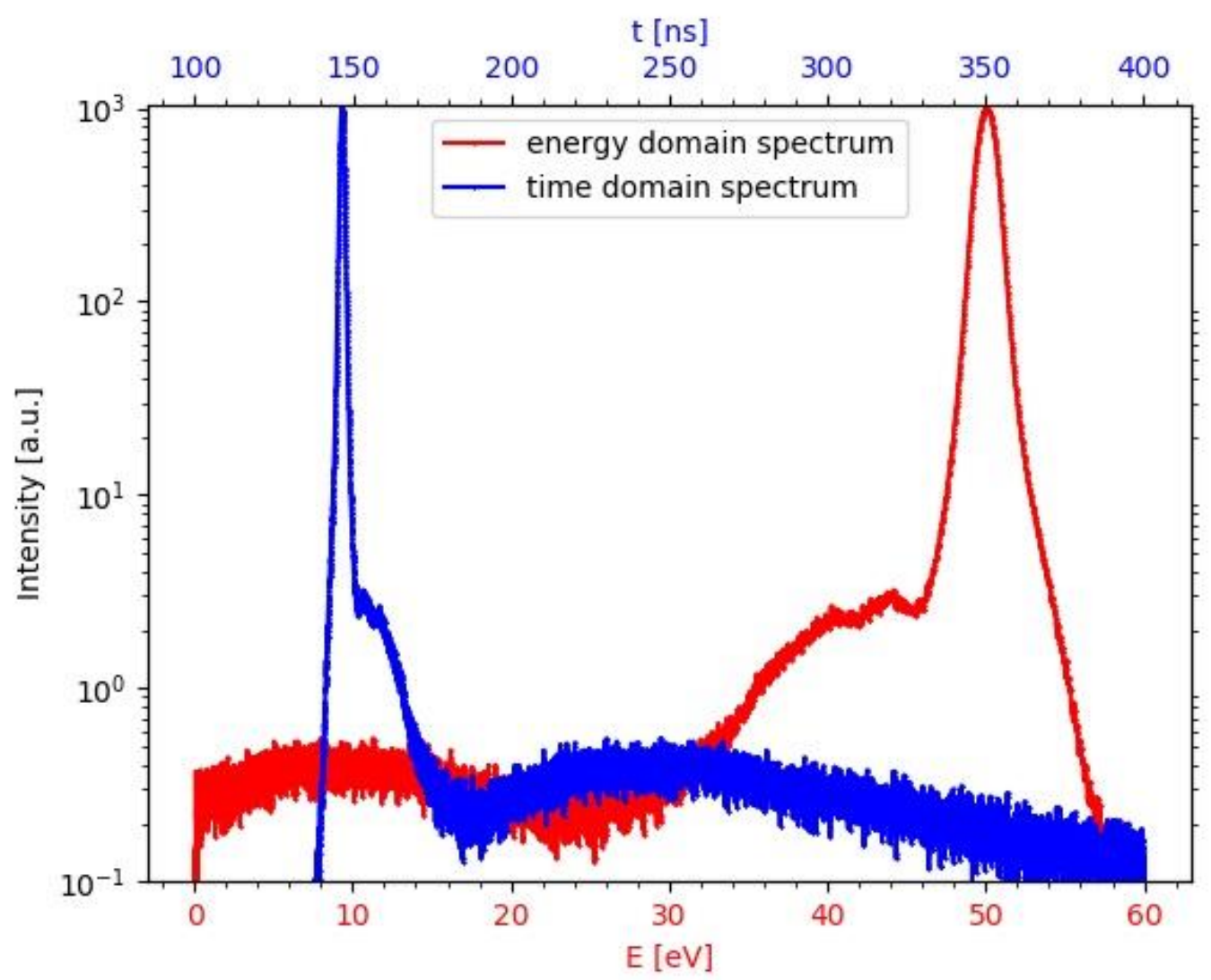

Figure 2. Measured ToF spectrum for graphene sample, landing energy EL $=50 \mathrm{eV}$; both time and energy domain are displayed.

References

[1] DIGUN, https://www.delong.cz/products/electron-guns/ (accessed February 24, 2020). 
[2] I Müllerová and L Frank, Adv. Imag. Elect. Phys. 128 (2003), p. 309-443.

[3] WE Stephens, Phys. Rev. 69 (1946), p. 691.

[4] RoentDek Delayline Detectors, www.roentdek.com/detectors/ (accessed February 24, 2020).

[5] MP Seah and WA Dench, Surf. Interface Anal. 1 (1979) p. 2-11.

[6] The authors acknowledge funding from the Technology Agency of the Czech Republic (Centre of Electron and Photonic Optics, no: TN01000008). 